\title{
Laparoscopic Lavage in Complicated Diverticulitis with Colonic Perforation, Always Be Closing?
}

\author{
Jasper Sijberden Heleen Snijders Susanna van Aalten \\ Department of Surgery, Groene Hart Hospital, Gouda, The Netherlands
}

\section{Keywords}

Colonic diverticulitis · Perforation · Purulent peritonitis · Laparoscopic lavage · Surgical technique

\begin{abstract}
Laparoscopic lavage is seen as an acceptable alternative to colonic resection in selected patients with acute diverticulitis with purulent peritonitis. There is no consensus on what surgical technique should be used when performing this procedure. This case series describes the disease course of 3 patients with acute diverticulitis with purulent peritonitis treated with laparoscopic lavage and direct suturing of a colonic perforation. All patients (38- and 71-yearold males and a 44-year-old female) were seen in the emergency department due to acute lower abdominal pain. Clinical examination and laboratory and imaging studies were suggestive of perforated diverticular disease. Laparoscopic lavage with placement of drain(s) and direct suturing of a colonic perforation was performed. Postoperative treatment with intravenous antibiotics was continued for a variable term. Postoperative courses were uneventful. Patients were discharged on postoperative days 5, 5, and 7. At almost 1-year follow-up, all patients are in good clinical condition and have not had a recurrent episode of diverticulitis. Therefore, this case series shows promising results of laparoscopic lavage with direct suturing of colonic perforation in patients with diverticulitis with perforation and purulent peritonitis.

(C) 2021 The Author(s).

Published by S. Karger AG, Basel
\end{abstract}

\section{Introduction}

Colonic diverticular disease is a disease entity which entails the protrusion of mucosa and submucosa through the muscular layer of the colon. The protrusions which are formed are called diverticula [1]. Diverticular disease is often an asymptomatic disease which mainly affects the Western elderly ( $>50$ years of age) $[1,2]$. Diverticula are most commonly found in 
Sijberden et al.: Direct Suturing of Colonic Perforation When Performing Laparoscopic Lavage

Table 1. Modified Hinchey classification for diverticulitis [9]

\begin{tabular}{ll}
\hline Modified Hinchey classification \\
\hline 0 & Mild diverticulitis, diagnosis based on clinical presentation \\
Ia & Limited pericolic inflammatory process \\
Ib & Limited pericolic abscess \\
II & Pelvic, intra-abdominal, or retroperitoneal abscess \\
III & Generalized purulent peritonitis \\
IV & Fecal peritonitis \\
\hline
\end{tabular}

the sigmoid colon [1]. In acute diverticulitis, one or more diverticula are inflamed. Severity of disease in acute diverticulitis can be defined using the Hinchey classification (Table 1). In Hinchey 3 and 4 diverticulitis, there is a free colonic perforation, which leads to a generalized purulent or fecal peritonitis. If clinical and radiological findings suggest this is the case, emergency surgical treatment is indicated [3].

Over the years, a wide range of surgical techniques have been used to treat patients with Hinchey 3 or 4 diverticulitis [4]. Recently, after publication of the SCANDIV [5], DILALA [6], and LADIES [7] trials, laparoscopic lavage is seen as an alternative to resection of the diseased part of the colon in selected patients with Hinchey 3 diverticulitis (purulent peritonitis). The Dutch acute diverticulitis guideline states that "laparoscopic lavage and drainage is a treatment modality which can be considered as an alternative to resection in these patients, but that one must take the clinical condition, patient's characteristics, expertise of the surgeon, and patient's wishes into account. It also states, "the surgeon must consider not to remove or manipulate the infiltrate to prevent a sealed-off perforation from becoming a free perforation" [8]. The guideline refers to the 3 earlier mentioned trials which all state that extensive dissection of adhesions should not be performed. None of these studies describe evidence to support this advice.

The aim of this study is to describe a case series of 3 patients with clinical and radiological signs of a free perforation treated with laparoscopic lavage and drainage with closure of the colonic perforation. The patients included in this series were asked for informed consent to describe their disease history including peroperatively taken pictures, to which all patients agreed.

\section{Case Series}

Case A

A 44-year-old female with no past medical history was referred to the gynecology department of our hospital because of progressive lower abdominal pain more left than right in the last 2 days. The patient had no other complaints except for a subfebrile temperature of $38^{\circ} \mathrm{C}$. Cervical motion tenderness was present. Transvaginal echography showed a simple cyst of the left adnexa and a minimal amount of free fluid in the pouch of Douglas. No gynecological cause for the complaints could be identified. Surgery was consulted. We observed a painful looking patient. Vital signs were normal. Physical examination of the abdomen revealed hypoactive bowel sounds and tenderness of the whole abdomen, mostly in the lower abdomen more left than right. Laboratory studies showed leukocytosis $(14,300$ cells $/ \mu \mathrm{L})$ and an elevated C-reactive protein $(59 \mathrm{mg} / \mathrm{L})$. Abdominal ultrasound revealed a circumferential thickening of the wall of the sigmoid colon with possible stranding of the pericolic fat. Since no complete overview of the pelvis could be obtained, an abdominal CT was performed. CT

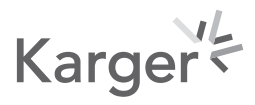




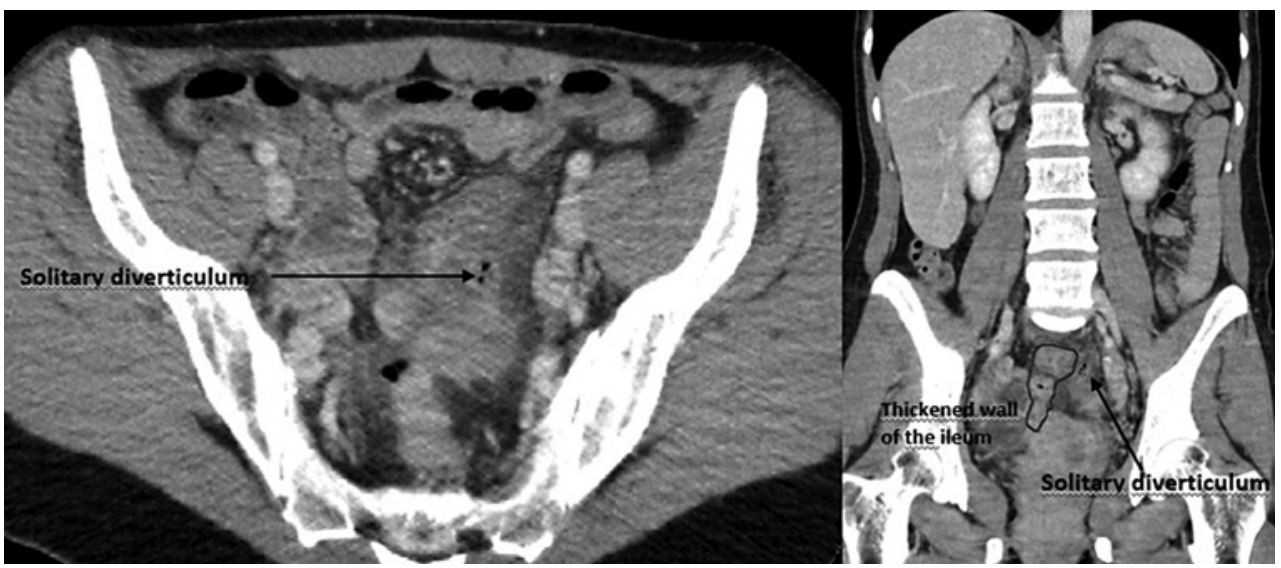

Fig. 1. Abdominal CT images of case A showing a solitary perforated diverticulum of the sigmoid colon and a thickened wall of the ileum nearby.

showed fat stranding around the sigmoid colon and extraluminal air nearby a solitary diverticulum (Fig. 1). The radiological diagnosis of acute diverticulitis with a free perforation was made.

Emergency laparoscopy was performed which showed acute diverticulitis of the sigmoid colon with a substantial amount of pus in the pelvis and signs of an intramural perforation (incomplete perforation where not all layers of the colonic wall are perforated). After extensive lavage, the perforation was closed using the epiploic appendages and a Vicryl ${ }^{\circledR}$ suture. A drain was left behind in the pouch of Douglas. Postoperatively, the patient was treated with cefuroxime and metronidazole intravenously. The drain was not productive anymore after 4 days and was removed. After 5 days of intravenous antibiotic treatment, the patient was discharged in good clinical condition. Follow-up in the outpatient clinic has been uneventful up till now (10 months after surgery).

Case B

A 71-year-old male with a past medical history of an earlier episode of acute diverticulitis of the sigmoid colon with an abscess treated with intravenous antibiotics was referred to the emergency department with acute lower abdominal pain. The patient described progressive lower abdominal pain with decreased appetite, which acutely started on the morning of presentation. An ill and painful looking patient was seen. Vital signs were normal. Physical examination of the abdomen revealed hypoactive bowel sounds, generalized abdominal guarding, and generalized tenderness most prominent in the left lower quadrant. Laboratory studies showed leukocytosis $(13,000$ cells $/ \mu \mathrm{L}$ ) and a CRP within normal range (3 mg/L).

An abdominal ultrasound and subsequently an abdominal CT were performed. Abdominal CT showed a small amount of free air near the gallbladder and fat stranding near the sigmoid colon (Fig. 2). The radiological diagnosis of acute diverticulitis of the sigmoid colon with a covered perforation was made. The patient was admitted. Because the clinical condition of the patient deteriorated the next day, with increased abdominal pain and CRP, a diagnostic laparoscopy was performed.

Pus was seen in the lower abdomen and above the liver, and an intramural perforation was seen in the proximal sigmoid (Fig. 2). Lavage was performed, and the perforation was closed by direct suturing with Vicryl 2.0. A drain was placed in the rectovesical pouch. Intravenous treatment with cefuroxime and metronidazole was started. On the third day postoperatively, the drain was removed, and antibiotic treatment was discontinued. On the fifth 


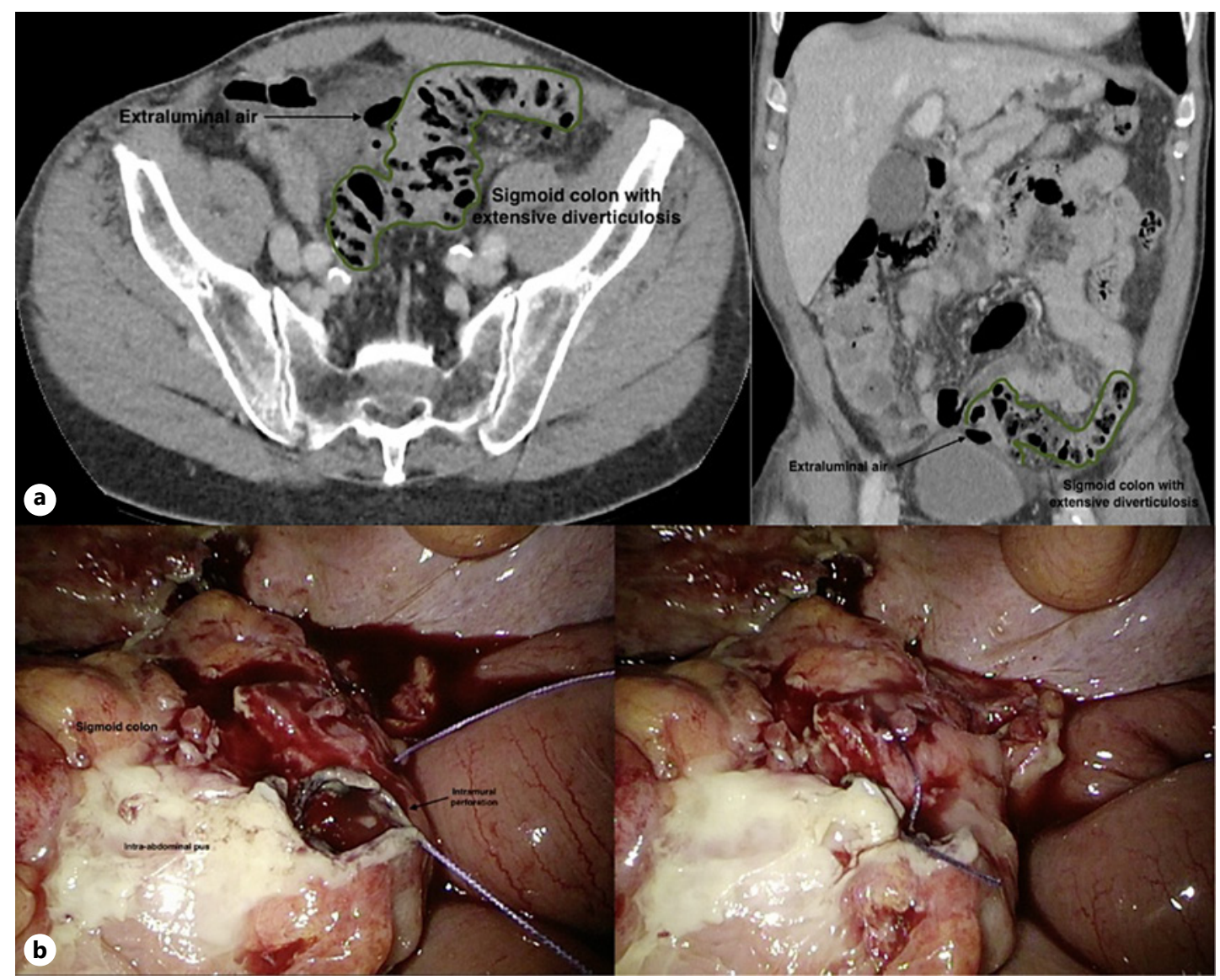

Fig. 2. a Abdominal CT images of case B showing extensive diverticulosis of the sigmoid colon, fat stranding, and extraluminal air just cranial of the bladder. $\mathbf{b}$ Laparoscopy images of case B showing sigmoid colon covered with pus; intramural perforation shown in the left picture is closed by direct suturing, and result is shown in the right picture.

day postoperatively, the patient was discharged. In the first months after surgery, the patient presented several times with colicky pain in the lower abdomen. Extensive workup (lab, ultrasound, CT, and colonoscopy) revealed no signs of recurrence of diverticular disease.

\section{Case C}

A 38-year-old male with no relevant past medical history was referred to the emergency department with acute abdominal pain. The patient described a changed defecation pattern in the last month and cramping abdominal pain during 1 week with acute worsening the morning of presentation. Since that morning, the pain was mainly localized in the right lower quadrant. A painful looking patient was seen, and vital signs were normal. Physical examination revealed abdominal guarding and tenderness of the lower abdomen.

Laboratory studies showed leukocytosis (13,300 cells/ $\mu \mathrm{L})$ and an elevated CRP (51 $\mathrm{mg} / \mathrm{L}$ ). Abdominal CT showed diverticulosis of the sigmoid colon with fat stranding, paracolic free air and free air ventral of the liver, and free fluid in the pelvis (Fig. 3). The radiological diagnosis of acute diverticulitis of the sigmoid colon with perforation was made. The patient was admitted, and antibiotic treatment with cefuroxime/metronidazole was started. Emergency laparoscopy was performed which showed pus in the pelvis and a perforation at the 


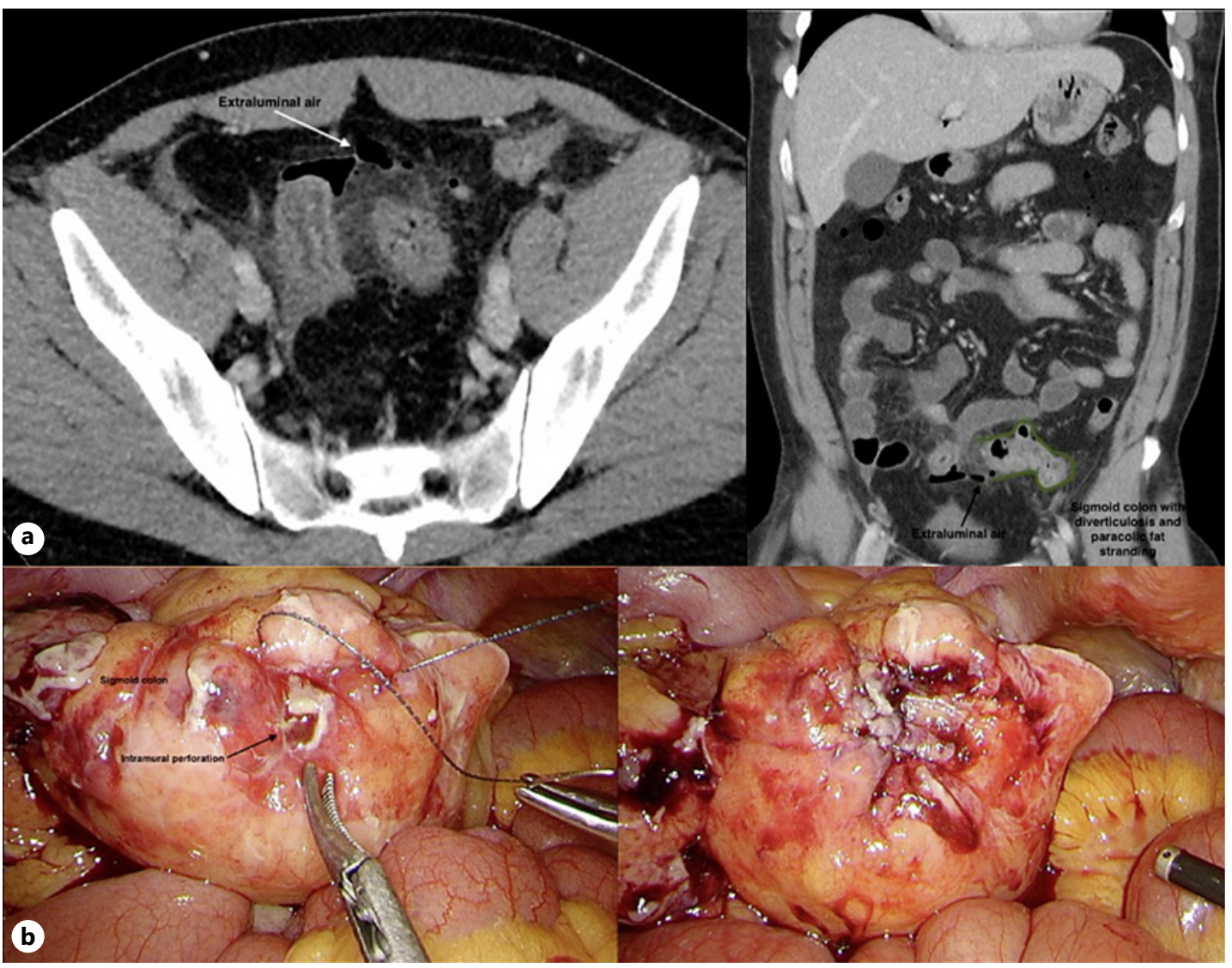

Fig. 3. a Abdominal CT images of case $\mathrm{C}$ showing diverticulosis of the sigmoid colon, paracolic fat stranding, and extraluminal air. b Laparoscopy images of case $\mathrm{C}$ showing sigmoid colon with intramural perforation which is closed by direct suturing with V-Loc.

mesenterial side of the sigmoid colon. Lavage was performed, and the perforation was closed by direct suturing with V-Loc ${ }^{\circledR}$ (Fig. 3). Two drains were placed, one in the rectovesical pouch and another close to the closed perforation. On the second day postoperatively, the drain in the rectovesical pouch was removed, and on the third day, the other drain was removed. Intravenous antibiotics were continued until the 5 day postoperatively and then switched to oral amoxicillin/clavulanic acid. The patient was discharged on the seventh day postoperatively in good clinical condition, and antibiotic treatment was continued orally so that patient received antibiotic treatment for a total of 10 days. Follow-up in the outpatient clinic has been uneventful up till now ( 9 months after surgery).

\section{Discussion}

This case series describes the clinical presentation, treatment, and disease course of 3 patients with diverticulitis with clinical and radiological signs of a free perforation treated with laparoscopic lavage and direct suturing of the peroperatively visualized colonic perforation in our center. Currently, there is no consensus on what exact surgical technique should be used when performing laparoscopic lavage [2]. While the Dutch guideline on acute diverticulitis discourages manipulation of the infiltrate [8], the surgeons in our center decided to close peroperatively visualized perforations. 
In recent publications, some authors plead for extensive adhesiolysis and colonic mobilization, and others believe that only limited adhesiolysis should be performed to possibly prevent a covered perforation from becoming a free perforation. In the case of peroperative detection of a free perforation, some authors view this as an absolute indication to perform colonic resection [2].

Others describe performing a colporrhaphy with direct suturing (with absorbable sutures covered by fibrin glue) combined with omentoplasty or only performing omentoplasty. An epiploic appendix can also be used to cover up the colonic defect [2]. The positioning and amount of drains also varies in the literature: some place a single drain close to the perforation or inflamed part of the colon, others only place a drain in the pouch of Douglas, and a third group of authors place multiple drains and, for example, position them in both the forementioned localizations. A small number of authors describe not placing a drain [2]. The study protocol for the earlier mentioned SCANDIV trial [5] "precluded dissection of inflammatory adhesions to the sigmoid colon." In the DILALA [6] and LADIES [7] trials, it is not described if the operative technique entailed adhesiolysis. It is clear though that in neither of these 3 trials suturing of a peroperatively visualized perforation was performed. Reoperation rates, at short time follow-up after index surgery, in the SCANDIV [5] and DILALA [6] trials were 20 and 13.2\%, respectively. Most of these reoperations were performed because of ongoing peritonitis and/or inadequately drained intra-abdominal abscesses. The question is if these reoperations could have partly been prevented by closing peroperatively visualized perforations and a different positioning of drain(s) placed during index surgery.

Our hypothesis is that the combination of extensive lavage and closure of the perforation leads to a larger degree of source control. If this is actually the case cannot be stated based on this very small case series, although clinical outcomes were promising. Further, preferably prospective, studies should be performed on what operative technique should be used when performing laparoscopic lavage. Of course patient characteristics, clinical condition, and extent of disease should be taken into account when choosing an operative strategy.

\section{Acknowledgment}

The authors thank Dr. M. Kulawska for her help in interpreting the CT scans.

\section{Statement of Ethics}

Written informed consent was obtained from the patients for publication of this case series and any accompanying images. After consulting the research specialist of the Groene Hart hospital, the conclusion was drawn that no approval of an institutional review body was needed.

\section{Conflict of Interest Statement}

The authors of this manuscript do not have any conflicts of interest to declare.

\section{Funding Sources}

This research did not receive any specific grant from funding agencies in the public, commercial, or not-for-profit sectors.

\section{Karger'}


Sijberden et al.: Direct Suturing of Colonic Perforation When Performing Laparoscopic Lavage

\section{Author Contributions}

Jasper Sijberden was involved in design of the work, drafting the manuscript, and final approval and agreed to be accountable for all aspects of the work. Heleen Snijders was involved in design of the work, revising the manuscript critically, and final approval and agreed to be accountable for all aspects of the work. Susanna van Aalten was involved in design of the work, revising the manuscript critically, and final approval and agreed to be accountable for all aspects of the work.

\section{Data Availability Statement}

Data sharing not applicable to this article as no datasets were generated or analysed during the current study.

\section{References}

1 Delvaux M. Diverticular disease of the colon in Europe: epidemiology, impact on citizen health and prevention. Aliment Pharmacol Ther. 2003;18(Suppl 3):71-4.

2 Gregori M, Cassini D, Depalma N, Miccini M, Manoochehri F, Baldazzi GA. Laparoscopic lavage and drainage for Hinchey III diverticulitis: review of technical aspect. Updates Surg. 2019 Jun;71(2):237-46.

3 Hinchey EJ, Schaal PG, Richards GK. Treatment of perforated diverticular disease of the colon. Adv Surg. 1978; 12:85-109.

4 Cirocchi R, Afshar S, Di Saverio S, Popivanov G, De Sol A, Gubbiotti F, et al. A historical review of surgery for peritonitis secondary to acute colonic diverticulitis: from Lockhart-Mummery to evidence-based medicine. World J Emerg Surg. 2017 Mar 9;12:14.

5 Schultz JK, Wallon C, Blecic L, Forsmo H, Folkesson J, Buchwald P, et al. One-year results of the SCANDIV randomized clinical trial of laparoscopic lavage versus primary resection for acute perforated diverticulitis. Br J Surg. 2017 Sep;104(10):1382-92.

6 Kohl A, Rosenberg J, Bock D, Bisgaard T, Skullman S, Thornell A, et al. Two-year results of the randomized clinical trial DILALA comparing laparoscopic lavage with resection as treatment for perforated diverticulitis. Br J Surg. 2018 Aug;105(9):1128-34.

7 Lambrichts DPV, Vennix S, Musters GD, Mulder IM, Swank HA, Hoofwijk AJM, et al. Hartmann's procedure versus sigmoidectomy with primary anastomosis for perforated diverticulitis with purulent or faecal peritonitis (LADIES): a multicentre, parallel-group, randomised, open-label, superiority trial. Lancet Gastroenterol Hepatol. 2019 Aug;4(8):599-610.

8 Dutch Federation of Medical Specialists. Guideline Acute diverticulitis. Last revision 2018 May 9. Available from: https://richtlijnendatabase.nl/richtlijn/acute_diverticulitis/startpagina_-_acute_diverticulitis.html. Consulted 2020 Mar 23.

9 Draaisma WA, van de Wall BJM, Vermeulen J, Unlu C, de Korte N, Swank E. Geen goed onderzochte behandeling voor diverticulitis. Ned Tijdschr Geneeskd. 2009;153:A648. 